\title{
Introduction to Cellular Drug Delivery
}

\author{
Present Realities and Future Prospects
}

\section{Kinam Park}

\section{Introduction}

Drugs play a central role in modern medicine (1). The total global pharmaceutical market in 2002 was more than $\$ 400$ billion, and the annual growth rate of the global market has been more than $10 \%$. The large market size allows big pharmaceutical companies to invest heavily in research and development (R\&D). In 2002 alone, the combined R\&D budget of Pfizer, Pharmacia, Merck, Eli Lilly, and Bristol-Myers Squibb was more than $\$ 16$ billion. Despite an ever-increasing R\&D budget, development of new drugs has been slow, and the number of new drugs introduced has become smaller than the number of drugs that went off patent protection. The real cost of developing a new drug is not well established, but it is generally agreed to be about $\$ 800$ million. One of the reasons for such a high cost is that many drug candidates are abandoned owing to their unfavorable physicochemical and biochemical properties. It is also becoming more and more difficult to develop new drugs due to the increasing difficulty of reaching their intended targets. One of the ways of making drug development more efficient is to utilize drug-delivery technologies from the early stages onward. The development of new drug-delivery technologies also makes the existing drug more useful.

Drug-delivery technologies contribute significantly in various stages of drug development. For a drug to exert its bioactivity, it has to enter the target cells. This seemingly simple process requires a series of steps that are often difficult to overcome. The steps of drug delivery to the target cells are described in Fig. 1. A drug first has to dissolve in aqueous solution first to have any chance of being bioactive (Step A in Fig. 1). Many newly developed drug candidates are poorly water soluble so a large number of potentially useful drugs are abandoned. Poorly soluble drugs can be dissolved in aqueous solution via polymeric micelles, polymeric nano/micro-particles, or polymeric prodrugs. New protein drugs can increase their bioactivity by protecting themselves in blood using various drug delivery technologies, e.g., PEGylation and microencapsulation. Once a drug is in solution, it has to be delivered to the target cells (Step B in Fig. 1). Commonly, a drug is delivered throughout the body, which causes unwanted side effects. Targeting minimizes such side effects. Targeting may be the only way for some treatments. For example, whether gene therapy will be successful or not depends entirely on whether genes can be delivered to the target site and effectively enter the 


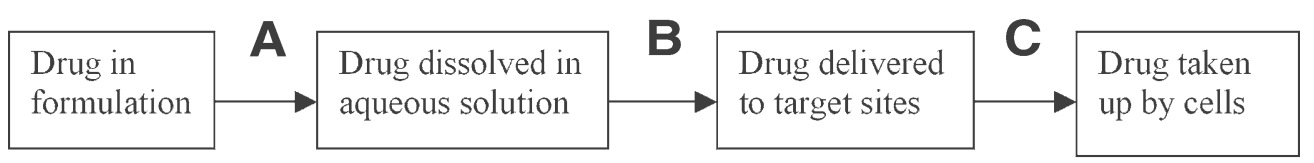

Fig. 1. Drug molecules in different stages.

target cells for expression (Step C in Fig. 1). Entering into the target cell is half of the process. The genes also must escape from endosomes to make themselves available for expression. For most drugs, the ultimate bioactivity is appreciated after they have entered the cells, and thus, cellular uptake can be considered one of the most important steps in drug delivery. In this regard, cellular drug delivery is a very timely topic to consider.

\section{Current Technologies in Cellular Drug Delivery}

There is a saying in China that you win every battle if you know both your enemy and yourself. Knowing one's enemy is critical in winning the war. In the war against disease, we have to know the enemy, such as viruses and bacteria. At the same time, we have to know ourselves, that is, the cells in our body. This book is about understanding ourselves in preparing for the war against diseases. We have to understand how our body functions and then apply that knowledge in the design of new strategies for efficient drug delivery.

Cellular Drug Delivery: Principles and Practice provides information on both drug uptake into cells and methods of improving cellular uptake. As listed in Table 1, the book discusses the cellular processes, drug delivery to cells, and targeting and formulation. The first three chapters provide background on how cells uptake nutrients and drugs, and other basic information on cell biology. We have a good understanding of transport processes through cell membranes that can be exploited in cellular delivery of various drugs (see Chapters 1 and 2). Understanding the biological processes of cellmediated responses (see Chapter 3) should provide strategies for promoting cell-mediated immunity against viral infection, such as influenza virus and, it is hoped, the virus causing severe acute respiratory syndrome (SARS). Two chapters are dedicated to nonviral DNA-delivery systems (see Chapters 4 and 5). Although viral vectors are most effective in gene delivery, the inherent danger of using live viruses has been proven to be problematic. There is no doubt that development of nonviral vectors will be essential in routine clinical applications of gene therapy in the future. One of the approaches for efficient cellular delivery may be utilizing naturally occurring peptides that are known to enhance entry into cell membranes (see Chapter 6). Transporters not only absorb drugs and nutrients into cells, but also they can efflux absorbed drugs, and thus understanding various transporters in cell membranes is important in optimizing drug therapy (see Chapters 7 and 8). Because the majority of drugs are delivered by oral administration, understanding the roles of various transporters in intestinal drug absorption is critical in screening various drugs (see Chapter 9).

The two chapters on targeting using colloidal carriers and protein nanocomplexes should be useful for the delivery of various types of drugs (see Chapters 10 and 11). The last six chapters discuss formulation-related problems, which often, unfortunately, are neglected, for without proper formulation, no drugs would be clinically useful. Bulk manufacturing of polynucleotides in their stable forms in effective delivery sys- 
Table 1

\section{Organization of the Book}

\begin{tabular}{ll}
\hline Topic & Chapter \\
\hline Cell biology & 1. Transport processes through cell membranes \\
& 2. Cell growth and cell cycle \\
3. Cell-mediated immunity \\
Cellular delivery & 4. Nonviral DNA delivery \\
& 5. Cellular delivery of nucleic acid \\
& 6. Membrane permeation promoting peptides \\
& 7. Transporters \\
8. MDR modulators (inhibitors) \\
9. Intestinal cell permeability \\
Formulation & 10. Colloidal carriers \\
& 11. Protein complexes \\
& 12. Preservation of DNA integrity \\
& 13. Cationic microparticles \\
14. Transdermal delivery \\
15. Immunoglobulin formulation \\
16. Antibody formulation for cancer treatment \\
17. Pharmaceutical profiling
\end{tabular}

tems is essential in widespread use of nucleotide-based therapeutics (see Chapters 12 and 13). One of the first applications in controlled drug-delivery technologies was the development of transdermal products, and one of the first therapies of antisense oligodeoxynucleotides may be by transdermal delivery (see Chapter 14). Immunoglobulins are considered to be good vectors to deliver T-cell epitopes to antigen-presenting cells (APCs), and targeted delivery of immunoglobulin-engineered microparticle systems have a great potential (see Chapter 15). Antibody-mediated targeted delivery of anticancer agents also holds great promise (see Chapter 16). Chapter 17 on the automated pharmaceutical profiling assays is one of the most interesting chapters of the book. Rapid screening of physicochemical and biochemical properties of drug candidates that affect cellular drug delivery provides new opportunities in pharmaceutical research beyond in vivo animal experiments.

\section{Opportunities in Cellular Delivery}

The concept of controlled drug delivery is more than four decades old, and the drugdelivery area is still evolving. Through the years, numerous novel drug-delivery systems have been developed and a large number of formulations are helping patients. Although the advances made to date are impressive, a number of breakthroughs are necessary for solving various problems facing us today. The main progress to be made is in the precision of targeting. Until a few decades ago, destroying a target in the enemy territory during a war required delivering hundreds of thousands of bombs that literally destroyed the whole town. Nowadays, laser-guided smart bombs or bombs with global positioning capability brought a new concept of one bomb per one target. The ultimate success in drug delivery is expected to bring about a concept of one drug 
molecule per one target. In fact, all that is required for successful gene therapy is to deliver a gene into a cell as long as the delivered gene is properly expressed. This means that the precision targeting has to be accompanied by effective cellular delivery.

For delivery of macromolecular drugs, such as anticancer drug molecules grafted to polymer chains, protein drugs, and genes, absorption into cells by endocytosis may be the most effective way. Endocytosis occurs when macromolecular drugs or drug carriers interact with the receptors on cell membranes, and receptor-mediated cellular uptake has been exploited quite extensively. In addition to receptor-mediated endocytosis, nonspecific electrostatic interaction with cell membranes is also known to cause endocytosis. One of the possible mechanisms of enhanced cellular absorption by cell penetration peptides $(2,3)$ and trans-activating transcriptional activator (TAT) (see Chapter 6) is nonspecific electrostatic interactions between positive charges in side chains of those peptides with cell membranes. Effective endocytosis based on nonspecific electrostatic interaction is important for designing the cellular delivery systems that also have the additional function of escaping from endosomes. Drug-delivery vehicles can be designed with high flexibility for effective endocytosis with a minimum number of specific ligand molecules that bind to receptors on cell membranes. Enhanced cellular delivery may also exploit the unusual ability of neutral block copolymers, such as poly(ethylene oxide)-poly(propylene oxide)-poly(ethylene oxide) triblock copolymers, to get inside cells. They are known also to decrease significantly the P-glycoprotein efflux activity in P-glycoprotein overexpressing membranes, probably through incorporating themselves into cell membranes, leading to increased fluidization (4). The cumulated data clearly demonstrated that those block copolymers are taken up inside cells to spread throughout the cytoplasm. Recent studies using fluorescent micelles showed that micelles made from polycaprolactone and poly(ethylene oxide) enter into cells by endocytosis to distribute themselves within several cytoplasmic compartments, but not in the nucleus (5). There is a great potential in improving cellular uptake using various polymeric micelles and in order to control the distribution of the absorbed drug in the cytoplasm.

Opportunity also exists in designing the delivery systems that have the ability to escape from endosomes once they enter cells by endocytosis. One of the main disadvantages of nonviral vectors as compared with viral vectors is their inability to deliver drugs intact from endosomes into the cytoplasm. A number of approaches have been attempted for providing endosome-disruptive properties. Endosome-disruptive hemagglutinin peptides from influenza virus and membrane-active amphipathic peptides have been used to release the delivered agents from endosomes to the cytoplasm $(6,7)$. Although these approaches are promising, they suffer from the shortage of such peptides. One recently developed approach is to utilize synthetic polymers that can disrupt the endosomal membranes at $\mathrm{pH}$ around 6.0. Polymeric histidine has shown its ability to disrupt endosomes when $\mathrm{pH}$ is lowered to deliver drugs into cytosol (8-10). Combining polycationic nature for endocytosis and polyhistidine for endosomal escape may be a practical combination for enhanced cellular delivery of a variety of drugs ranging from low molecular-weight anticancer drugs to proteins and genes.

Understanding cell biology, membrane interfacial phenomena, protein chemistry, and polymer chemistry are all essential in developing effective cellular delivery systems. Being an expert on any of these areas provides a great potential to open new avenues of cellular drug delivery. If the history of the pharmaceutical industry is any guide, one can easily imagine the ever-increasing values of such research areas and the 
high rewards accompanying them. Opportunities in the future basically are infinite and only our imagination is a limit. Cellular Drug Delivery: Principles and Practice will certainly serve as an excellent resource in expanding our imagination in developing novel drug-delivery systems for the most efficient methods of cellular delivery.

\section{References}

1. Drews J. In Quest of Tomorrow's Medicines. New York, NY, Springer-Verlag, 1999.

2. Langel Ü. Cell-Penetrating Peptides. Processes and Applications. Boca Raton, FL, CRC Press, 2002.

3. Nielsen PE. Peptide Nucleic Acids. Methods and Protocols. Totowa, NJ, Humana Press, 2002.

4. Batrakova EV, Li S, Alakhov VY, et al. Optimal structure requirements for Pluronic block copolymers in modifying P-glycoprotein drug efflux transporter activity in bovine brain microvessel endothelial cells. J Pharmacol Exp Ther 2003;304:845-854.

5. Savic R, Luo L, Eisenberg A, Maysinger D. Micellar nanocontainers distribute to defined cytoplasmic organelles. Science 2003;300:615-618.

6. Wagner E, Curiel D, Cotten M. Delivery of drugs, proteins and genes into cells using transferrin as a ligand for receptor-mediated endocytosis. Adv Drug Del Rev 1994;14:113-135.

7. Plank C, Zauner W, Wagner E. Application of membrane-active peptides for drug and gene delivery across cellular membranes. Adv Drug Del Rev 1998;34:21-35.

8. Midoux P, Monsigny M. Efficent gene transfer by histidylated polylysine/pDNA complexes. Biconjug Chem 1999;10:406-411.

9. Putnam D, Gentry CA, Pack DW, Langer R. Polymer-based gene delivery with low cytotoxicity by a unique balance of side-chain termini. Proc Natl Acad Sci USA 2001;98:1200-1205.

10. Benns JM, Choi JS, Mahato RI, et al. pH-sensitive cationic polymer gene delivery vehicle: $\mathrm{N}$ Ac-poly(L-histidine)-graft-poly(L-lysine) comb shaped polymer. Bioconjug Chem 2000;11:637-645. 